\title{
Transformationen im Online-Medienmarkt: Was wird aus den journalistischen Kompetenzen?
}

\author{
Michael Haller
}

Keywords: Informationsjournalismus, Journalistische Unabhängigkeit, Medienwandel, Kompetenzen, Kommerzialisierung, Funktionalismus, Normativität, Öffentlich-Rechtlich, Plattformen

\section{Abstract}

Ein Blick auf die Aus- und Weiterbildungsangebote zeigt den enormen Anpassungszwang, dem der Journalismus unter den Produktionsbedingungen im Internet ausgesetzt ist. Der hohe Wettbewerbsdruck und die unzureichende Gegenfinanzierung führen zum Verschleiß der tradierten Kompetenzen. Dieser Beitrag rekonstruiert die mit der Demokratietheorie begründeten Kompetenzgehalte, weiter die mit der universitären Journalist*innenausbildung verbundene Fehlentwicklung des Kompetenzbegriffs und diskutiert die beiden Perspektiven, die sich für die Entfaltung eines substanziellen Kompetenzverständnisses in der Welt der Onlinemedien bieten: Aussichten auf eine Kommunikationsgesellschaft, die über die Vorgänge in der Welt zuverlässig informiert werden und die damit verbundenen Beurteilungen und Positionen im öffentlichen Diskurs klären will.

Michael Haller: Transformationen im Online-Medienmarkt: Was wird aus den journalistischen Kompetenzen? In: Nils S. Borchers, Selma Güney, Uwe Krüger und Kerem Schamberger (Hrsg.): Transformation der Medien Medien der Transformation. Verhandlungen des Netzwerks Kritische Kommunikationswissenschaft. Frankfurt am Main: Westend 2021. DOI: https:// doi.org/10.53291/GCAL8505.

Prof. em. Dr. Michael Haller I Europäisches Institut für Journalismus- und Kommunikationswissenschaft (EIJK)। haller@uni-leipzig.de 
Wie und warum der Journalismus - man denkt an den aktuellen Informationsjournalismus - in einer Strukturkrise steckt, wird seit Jahren vielstimmig diskutiert. Die häufigste Erklärung lautet: Es ist das Internet. Wortführende Onlinejournalist*innen ${ }^{1}$ wie auch zahlreiche Kommunikations- und Medienwissenschaftler*innen (beispielsweise Neuberger 2002; Neuberger et al. 2009; Quandt 2005) haben unermüdlich auf die mit der Digitalisierung verbundenen Transformationsprozesse und auf die für die Onlinewelt neue »medienökonomische Wertschöpfungsdiskussion « (Kolo et al. 2012, 15-16) verwiesen. Die Modernisierer monierten, der Journalismus sei seinem überkommenen Rollenselbstverständnis als Gatekeeper und Welterklärer verhaftet geblieben und habe den Mediennutzungswandel des Publikums nicht ernst genommen oder nicht verstanden. Nun müsse er quasi nachsitzen und seine Hausaufgaben machen (Oswald 2013, 66-79), also den Transformationsprozess verstehen lernen, ihn akzeptieren und mitgestalten: »Wir sind mitten in einem Generationenwechsel - vom Schreiber hin zum Manager.« (Bönisch 2019, 60) ${ }^{2}$

Diese Sichtweise der Modernisierer hat sich zum Common Sense der Branche verfestigt. Man trifft sie heute in praktisch allen Einrichtungen der Aus- und Weiterbildung mit ihren einschlägigen Nachhol- und Umlernkursen etwa für Content-Marketing, Social-Media-Management oder digitales Storytelling für Produktmagazine, PR- und Werbekunden - stets mit dem Label »Journalismus « ${ }^{3}$ versehen. Eine Erhebung unter österreichischen Ausbildungsstätten bestätigt, dass in der Journalist*innenausbildung am weitaus häufigsten fachlich enge Kursthemen zu »Technik / Tools für Bildbearbeitung / Audio-, Videoschnitt« genutzt werden (Schätz und Kirchhoff 2020). Auch in Deutschland

1 Stellvertretend Plöchinger (2013) sowie sieben Jahre später Elmer et al. (2020).

2 Julia Bönisch, damals Mitglied der Chefredaktion der Süddeutschen Zeitung, schrieb in ihrem Beitrag, dass auch die Trennung zwischen Verlag und Redaktion nicht mehr zeitgemäß sei. Dem widersprach die SZ-Chefredaktion vehement (o. A. 2019).

3 Vgl. aktuelle Seminarangebote auf den Webseiten einschlägiger Weiterbildungseinrichtungen, Hier drei Beispiele: https://www.akademie-fuer-publizistik.de/ seminare/alle-seminare/online-seminar-in-drei-schritten-zu-mehr-reichweite; https://www.procontent.de/seminar/digitales-storytelling-interaktive-geschichten-fuer-online-und-mobile/; https://www.merkur.de/lokales/muenchen/stadt-muenchen/deutsche-journalisten-akademie-13794026.html. 
wird der Kompetenzumbau vorangetrieben, vorneweg die SpringerJournalistenschule, die im Herbst 2020 in die FreeTech-Academy der Axel Springer SE integriert wurde; deren »Ziel ist es, die eigene Technologie-Kompetenz langfristig auszubauen «. ${ }^{4}$

Mit diesen Stichworten möchte ich die mit dem interaktiven Web 2.0 verbundenen Techniken keineswegs in Frage stellen. Dass Journalist*innen auch in handwerklicher Hinsicht die vielfältigen Interaktionsmöglichkeiten der Onlinekommunikation beherrschen sollten, steht außer Frage. Zu diskutieren wäre indessen das von den Aus- und Fortbildungsprogrammen vermittelte Berufsverständnis. Denn ihnen gemeinsam ist die Auffassung, dass die journalistischen Kompetenzen an die Marktbedingungen der Onlinemedien - etwa: Reichweite statt Relevanz im Kontext der noch immer vorherrschenden »Gratiskultur $^{5}$ - angepasst und »transformiert « werden sollen (offenbar wird »Kompetenz« als abhängige Variable der »digitalen Transformation« definiert). ${ }^{6}$ Den Medienmacher*innen geht es nicht darum, öffentlich Kritik und Kontrolle zu üben und politische Vorgänge unter den Gegebenheiten des Internets aufzuklären; das Lernziel gilt der Rückgewinnung von Marktanteilen vermittels der die Onlinewelt beherrschenden »Aufmerksamkeitsökonomie« (Ruß-Mohl 2017, 47-48). Und so lautet die von Medienmacher*innen alljährlich auf der re:publica, der Innovation Week von Media Lab Bayern und der Hamburger nextMedia-Konferenz gestellte Frage: Kann der Informationsjournalismus seine Strukturkrise überwinden, indem er sich in der Onlinewelt den „Gesetzen der modernen Erregungsindustrie« (Pörksen 2018) fügt, die derzeit den Medienwandel dirigieren? ${ }^{7}$

Diese Frage unterstellt, dass eine neue, der Digitalisierung der Medien geschuldete Transformationsstrategie entwickelt werden müsse (Schätz und Kirchhoff, 2020). Meine erste These lautet dem gegenüber:

4 https://www.axelspringer.com/de/presseinformationen/axel-springer-gruendet-freetech-academy-und-investiert-in-den-ausbau-der-technologie-kompetenz?

5 https://de.wikipedia.org/wiki/Gratiskultur.

6 Im Zusammenhang der Digitalisierung und der Onlinemedien wird der Begriff "Transformation« inflationär gebraucht. Ich folge hier der etwas grobschlächtigen Wikipedia-Definition: »Die digitale Transformation bezeichnet einen fortlaufenden, in digitalen Technologien begründeten Veränderungsprozess, der in wirtschaftlicher Hinsicht speziell Unternehmen betrifft.« https://de.wikipedia. org/wiki/Digitale_Transformation.

7 Näheres hierzu unter: https://www.nextmedia-hamburg.de/jeff-jarvis-skizziert-beim-scoopcamp-2020-die-zukunft-der-medienbranche/. 
Das Projekt, Journalist*innen in Kommunikationsmanager*innen der digitalen Newsmedien zu verwandeln, stützt sich auf ein Kompetenzverständnis, das keineswegs neu ist, sondern im Fortgang der vergangenen 30 Jahre an den Hochschulen fundamentiert wurde und die Journalist*innenausbildung mehr und mehr beeinflusst hat. Wie erklärt sich das?

\section{2 Über die Funktionalisierung journalistischer Aufgaben}

Anders als in der DDR, wo aus ideologischen Gründen die Journalist*innenausbildung schon in den 1950er Jahren akademisiert und auf die Funktion des Transmissionsriemens der Staatspartei ausgerichtet wurde, blieb Journalismus in der westlichen Bürgergesellschaft ein von der individuellen Eignung abhängiger Anlernberuf ohne Zugangssperre und von daher ein Forum für Begabte und Berufene wie auch ein Spielfeld für Um- und Seiteneinsteiger. Erst im Fortgang der 1960er Jahre änderte sich dies.

Anlass gaben der wachsende Einfluss der reichweitestarken Rundfunksender auf das Meinungsklima, der aggressive Kampagnenjournalismus der Boulevardmedien, die politischen Wirkungen des aufkommenden Investigativjournalismus (sinnbildlich die sogenannte Spiegel-Affäre 1962) und schließlich die vom Bundesverfassungsgericht 1961 (Rundfunkurteil) und 1966 (Spiegel-Urteil) dem Journalismus zugewiesene »öffentliche Aufgabe« (BVerfGE 20, 162-230): Diese »Politisierung von Gesellschaft und Journalismus« (Donsbach 1999, 497) löste unter Medienkontrolleuren (Presserat), Medienmachern, Zeitungsverlegern und Kommunikationswissenschaftlern eine »Professionalisierungsdebatte« aus (Koszyk 1974; Langenbucher 1974; Hömberg 1978). Sie führte zu dem Entschluss, die geforderten Kompetenzen des Journalist*innenberufs konzeptionell zu fassen und sie für die damals vielfältigen Ausbildungswege als Zielstellung zu formulieren. Die vom Deutschen Journalisten-Verband (DJV) in seinen Publikationen umrissenen Sach- und Vermittlungskompetenzen waren damals vom elitenkritischen Zeitgeist beseelt (Zeuner 1973, 13-16; Donsbach 1982, 163-172). Sie wiesen dem Journalismus neben der Informationsfunktion die Aufgabe zu, aus unabhängiger Sicht gegenüber Staat, Parteien und Wirtschaft eine öffentliche Aufklärungsfunktion wahrzunehmen (Schmolke 1970, 54) - ein Rollenverständnis, das die »innere Presse- 
freiheit $«^{8}$ als Bedingung verstand. Rund drei Viertel der Journalist*innen - verschiedenen Befragungen zufolge - reklamierten damals diese Rolle für sich (Donsbach 1982, 173; Fabris 1971, 360-368).

Als dann im Fortgang der 1970er Jahre mehrere Universitäten Journalismus-Studiengänge einrichteten, veränderte sich das Kompetenzverständnis und damit auch das Ausbildungsziel der Journalistik. ${ }^{9}$ Der Hauptgrund: In jener Zeit gewann dort die vor allem von Niklas Luhmann ausgearbeitete Systemtheorie an Einfluss. Kommunikationsund Medienwissenschaftler*innen nutzten sie zur Begründung ihrer Kritik an den normativen, als »links« etikettierten Medien- und Diskurstheorien..$^{10}$ Das Reden über wertneutrale System- und Umweltfunktionen kam in Mode und prägte das Journalismusverständnis als »Funktionsbewusstsein « in den Kommunikations- und Medienwissenschaften (Weischenberg 1990, 33).

Für dieses akademische Funktionsverständnis steht pars pro toto der Leitsatz des Systemtheoretikers und Journalistik-Studiengangleiters Manfred Rühl: Die Journalist*innen hätten keine Aufklärungsund keine Diskursfunktion; die Primärfunktion des »Systems Journalismus « bestehe vielmehr in der »Herstellung und Bereitstellung von Themen zur öffentlichen Kommunikation « (Rühl 1980, 319). Andere der Systemtheorie zugeneigte Journalistik-Wissenschaftler*innen erkannten aber, dass diese ausgedachte Definition auch die weite Welt des Fiktionalen und des Entertainments einschließt, praktisch also unbrauchbar ist. In der Folge theoretisierten sie über andere, den Kompetenzbegriff determinierende Funktionen des Journalismus, nämlich »aktuelle« Themen oder im Sinne Luhmanns einfach nur »Informationen « bereitzustellen (mit dem neuen Problem der Abgrenzung etwa gegenüber dem synthetischen Output der Datenbanken wie auch der Informationsbeschaffung von Geheimagent*innen und Steuerfahnder*innen). ${ }^{11}$ Um eine mit dem Luhmann'schen Systemdenken kompatible Kompetenz-Definition kümmerte sich vor allem Siegfried

8 Ende der 1960er Jahre ging es um die Frage, wie sich die Unabhängigkeit der Zeitungsredaktion gegenüber ihrem Medieneigentümer sichern ließe (Skriver 1970).

9 Vgl. die synoptische Darstellung »Ausbildung in den Tageszeitungen« (Ausbildungsrichtlinien und Tarifvertrag) in DJV (1990, 71-83).

10 Es war ein Machtkampf um Einfluss und Deutungshoheit, gut beschrieben insbesondere bei Holzer (1973, 11-29).

$11 \mathrm{Zu}$ den Gründen für die Realitätsferne jenes akademischen Journalismusverständnisses siehe Haller (2000, 110-120; 2012) sowie Donsbach (2012, 31-33). 
Weischenberg, der für das Journalistikstudium folgende drei Kompetenzdimensionen elaborierte: Fachkompetenz (Wissen und Handwerk), Vermittlungskompetenz (Artikulationsfähigkeit, Präsentation und Darstellungsformen) sowie Sachkompetenz (Ressort- und Spezialwissen). Der gesellschaftspolitische Zusammenhang wurde ausgeklammert, die »soziale Orientierung « als »Funktionsbewusstsein« der Vermittlungskompetenz unterstellt (Weischenberg 1990, 24). Den Älteren unter uns dürfte die gesamtdeutsche Enquête der Münsteraner Forschergruppe Weischenberg, Altmeppen und Löffelholz (1994) noch in Erinnerung sein: Was kompetente Journalist*innen in West wie Ost auszeichne, wurde mit Hilfe einer Delphi-Expertenbefragung ermittelt und nach Maßgabe der Weischenberg'schen Matrix kanonisiert: Fachkompetenz, Vermittlungskompetenz, Sachkompetenz sowie soziale Orientierung. Dieses Set fand in viele Journalistik-Curricula westdeutscher Universitäten Eingang, nicht aber in die Leipziger Fakultät, die das normative Leitbild der demokratietheoretisch begründeten »öffentlichen Aufgabe« zur Maxime erhob. ${ }^{12}$ Im Vergleich zu den anderen dualen Diplomstudiengängen (Dortmund, Eichstätt) fand jener in deutschen Chefredaktionen die relativ größte Wertschätzung (Harnischmacher 2010, 221).

\section{Die Ausdifferenzierung der Kompetenz-Matrix}

Zweifellos half die Münsteraner Kompetenz-Matrix, die akademische Journalist*innenausbildung systematisch zu begründen und auf ein Fundament zu stellen, das die Industrialisierung der Aussagenproduktion rechtfertigt. Sie offenbarte allerdings eine dreifache Problematik: Erstens fällt die starke Betonung der instrumentellen Ebene (das »Fachlich-Sachliche«) auf, zweitens die Unterbewertung der Determinanten der Marktwirtschaft (Medienökonomie) sowie drittens die mangelnde Beschreibung demokratietheoretisch fundierter Funktionen (Normativität). Im Rückblick gewinnt man den Eindruck, dass die von Medienpraktiker*innen in den frühen 1970er Jahren progressiv entwickelten Kompetenz-Inhalte (Sachaufklärung, Meinungsvielfalt, Kritik und Kontrolle) von den systemtheoretisch denkenden Journalistik-Dozent*innen nicht gewollt, vielleicht auch nicht verstanden wurden. Jedenfalls

12 Das »Leipziger Modell« wurde vom Verfasser im Laufe der 1990er Jahre an Jahreskonferenzen des DJV und des BDZV wiederholt vorgestellt und diskutiert. 
haben sie die Münsteraner Matrix im Fortgang der akademischen Debatte - im Bemühen um Anpassung an den Medienwandel - immer weiter ausdifferenziert. So erweiterte Klaus Meier die vier KompetenzDimensionen um die Felder »Technik- und Gestaltungskompetenz «sowie »Organisations- und Konzeptionskompetenz (Abb. 1). Immerhin umschrieb er dieses Kompetenzbündel deutlich pragmatischer, auch näher an den normativ zu begründenden Handlungsmaximen:

Journalismus recherchiert, selektiert und präsentiert Themen, die neu, faktisch und relevant sind. Er stellt Öffentlichkeit her, indem er die Gesellschaft beobachtet, diese Beobachtung über periodische Medien einem Massenpublikum zur Verfügung stellt und dadurch eine gemeinsame Wirklichkeit konstruiert. Diese konstruierte Wirklichkeit bietet Orientierung in einer komplexen Welt. (Meier 2007, 13; unverändert in der 4. Auflage 2018, 14).

Gleichwohl hielt der Systemtheorie-Jargon die akademische Journalistik besetzt (in Meiers Beschreibung recherchieren, selektieren und präsentieren nicht die Akteur*innen, also die Journalist*innen, sondern das abstrakte System »Journalismus«).

Es ist hier nicht der Platz, die aus jener Kompetenzdebatte abgeleiteten Curricula mit ihren Ausbildungszielen und ihrer Praxistauglichkeit zu diskutieren..$^{13}$ Es soll der Hinweis genügen, dass die Idee des gesellschaftlichen Auftrags, aus unabhängiger Perspektive durch Kritik und Kontrolle die Voraussetzung für eine politisch aufgeklärte Öffentlichkeit zu schaffen, nicht zurückgeholt wurde; vielmehr verflüchtigte sich dieses Leitbild zugunsten operativer Fertigkeiten, die den industriellen Routinen der sich beschleunigenden Medienproduktion dienen (stellvertretend Oswald 2013, 63-80) und immer neue, auf den Medienkonsumentenmarkt zugeschnittene Spezifitäten generieren (beispielhaft Becker und Kaiser 2014 nach Maßgabe von www.medienwiki.org) .

Ich leite daraus meine zweite These ab, dass schon seit Beginn der 1990er Jahre in der Aus- und Weiterbildung des Journalismus die auf Marktgängigkeit ausgerichteten instrumentellen Fertigkeiten in den Fokus rückten und den Journalismus unter der Etikette »Transformation « auf Dienstleistungsfunktionen im Kontext kommerzialisierter Medienproduktionen verkürzten - rund zehn Jahre bevor sich mit dem

13 Beschreibungen finden sich im DJV-Memorandum von 2003 sowie im Buchteil III »Klassisches Handwerk und Medientraining « des Sammelbandes herausgegeben von Dernbach und Loosen (2012, 183-271). 


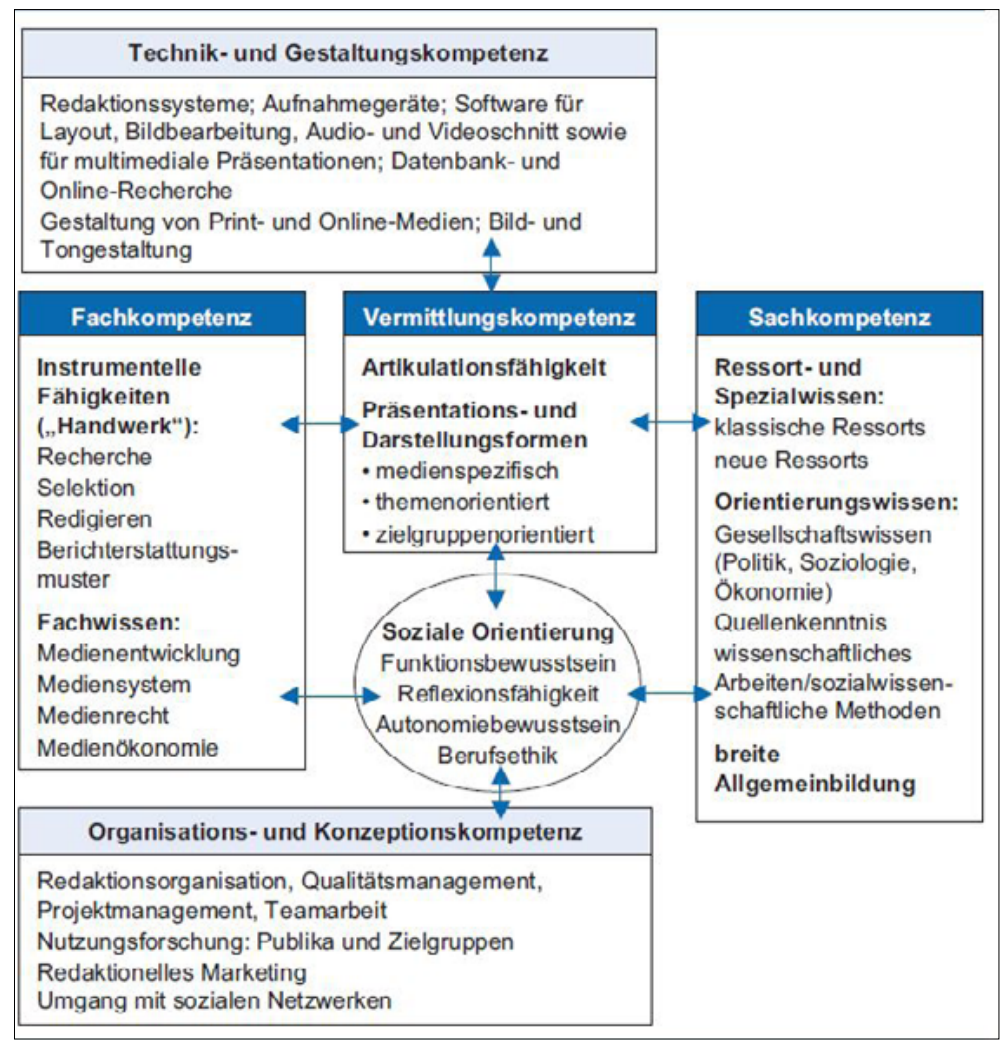

Abb. 1: Dimensionen journalistischer Kompetenz nach Weischenberg (1990) und Meier (2007).

Web 2.0 die Aufmerksamkeitsökonomie (»Gratis-Kultur«) durchzusetzen begann. Ist dieser Trend unumkehrbar?

\section{Die Umwelt als neue Dimension}

In der Zeit, als sich das Web 2.0 etablierte, führte die European Journalism Training Association (EJTA) in 16 europäischen Ländern eine Umfrage unter 402 Chef- und Ausbildungsredakteur*innen durch. ${ }^{14}$ Aus den Antworten konzipierte man im Jahr 2006 die sogenannte Tartu-

14 Der Dachverband EJTA umfasst 60 Hochschulen und Ausbildungsakademien mit Journalistik-Lehrgängen. 
Deklaration. ${ }^{15}$ Sie umfasst nicht sechs, sondern 50 in zehn Dimensionen gegliederte Kompetenzen, die für das journalistische Handeln, vor allem für redaktionelle Tätigkeiten maßgeblich sein sollen (»be able to ...«). Diese Kompetenz-Items veranschaulichen, wie vielgestaltig nun die Anforderungen gesehen wurden, die aus Sicht der Medienmacher*innen das journalistische Handeln auszeichnen. Auffällig dabei ist die unterschiedliche Gewichtung einzelner Kompetenzen zwischen Redaktionsleiter*innen und Ausbilder*innen, indem letztere deutlich stärker den gesellschaftlichen Bezug und die soziale Verantwortung für die Folgen journalistischen Handelns betonen (vgl. Baer et al. 2008). Umgekehrt legten die Redaktionschef*innen deutlich mehr Gewicht auf Fertigkeiten, die Managementwissen und technisches Know-how fokussieren und insofern der Medienökonomie in der Onlinewelt dienen.

Vor der Frage, ob mit der Interaktivität der Onlinekommunikation nicht auch eine Rückkehr zu den Kompetenzen angezeigt sei, die den personalisierten Interaktionen Rechnung tragen, mithin weniger systemstrukturell und mehr auf die Akteur*innen zentriert auszurichten seien, hat die Medienexpertin Alexandra Stark (2010) das EJTA-Kompetenzfeld vermittels der Dimensionen »Wissen/Können/Wollen« strukturiert. Bemerkenswert ist hier die Dimension »Wollen«, zu der Stark die Merkmale »Haltung/Einstellung/Bereitschaft« zählt. Es überrascht nicht, dass bei dieser Strukturierung das Kompetenzfeld »Können« (Schwerpunkt »handwerkliche Fertigkeiten«) erneut deutlich dominiert (Abb. 2). Gleichwohl steht dieser Ansatz für den Versuch, die journalistischen Kompetenzen aus der Technikfixierung zu lösen und in den Kontext des gesellschaftlichen Wandels zu stellen: Zur Menge der determinierenden Treiber der Transformation zählt sie neben dem technologischen und dem wirtschaftlichen Wandel auch die sich rasant ändernde Mediennutzung, die ihrerseits den Gesellschaftswandel prägt und insofern zur soziokulturellen Umwelt des Journalismus gehört. Auf diese Umwelt, so Starks Design, sollten sich inskünftig die dem individuellen »Wollen « zugeschriebenen Kompetenzen der TartuDeklaration beziehen, so vor allem die mit Innovation, Verantwortung und Diskursivität konnotierten Fertigkeiten (ebd., 65-67). Darin lässt sich der idealistische Wunsch erblicken, im Zeitalter des Web 2.0 die journalistischen Kompetenzen um soziale, interkulturelle und umweltbezogene Fertigkeiten zu erweitern (ebd., 62-63) - »Wunschdenken«

15 https://www.ejta.eu/tartu-declaration. 
(ebd., 66) deshalb, weil aus der berufsspezifischen Kompetenz ein universelles Vermögen werden soll, dessen Hauptqualität in der ununterbrochenen Anpassung an sich stetig verändernde Umwelten besteht: tranformatorisches Mitdenken und Handeln als Superkompetenz.

\section{5 Über alternative Kommunikationsräume und -ansprüche}

Dieser Rückblick auf vergangene Kompetenzdebatten sollte deutlich machen, dass mit der Professionalisierung des Berufs zunächst die politische Rolle des Journalismus im Mittelpunkt stand; es folgte die Debatte um die kompetenzbehindernden ökonomischen Rahmenbedingungen journalistischen Handelns, ehe die abstrakt-systemisch gedachte Funktionalität des Journalismus das Kompetenzverständnis prägte. Seit dem Web 2.0 und der Dominanz der Onlinemedien diffundiert der Begriff in immer mehr Merkmale und verliert sein auf den Journalist*innenberuf bezogenes Profil. Diese Diffusion ist reaktiv und insofern ein von den marktwirtschaftlichen Treibern des Medienwandels erzeugter Effekt: Die reichweitestärksten Newsmedien des Web 2.0 (im Pandemie-Jahr 2020 waren es die Online-Ausgaben von Bild, Spiegel und Focus) haben unter der einleitend erwähnten Etikette »digitale Transformation « den Journalismus instrumentalisiert und seine Kompetenzmerkmale auf die Vermarktungsinteressen zugeschnitten. Zudem sind auch die digitalen Kommunikationsräume, die der gesellschaftlichen Verständigung dienen, inzwischen weitgehend kommerzialisiert. Selbst der von derartigen Interessen freigehaltene öffentlichrechtliche Rundfunk beugte sich dem von den privaten Anbietern erzeugten Quotendruck durch Programmanpassungen (»Konvergenzhypothese«; Schatz 1994, 67-77; Merten 1994).

An dieser Stelle erwarte ich den Hinweis, dass viele mit »alternativ« apostrophierte Onlinemedien - beispielhaft in Deutschland das Regionalmedium Kontextwochenzeitung, in der Schweiz Republik und in den Niederlanden DeCorrespondent - als Ausbruch aus dieser Sackgasse zu deuten seien. Es stimmt, dass ihnen der Versuch gemeinsam ist, die tradierten, normativ begründeten Funktionszuschreibungen in die digitale Medienumwelt zu transferieren, also beispielsweise die informierende Sachaufklärung über relevante Vorgänge unter die Maximen Transparenz, Meinungsvielfalt und Diskursivität zu stellen. Doch bedürfen auch sie der Bezuschussung durch Mäzen*innen, Gönner *innen und 


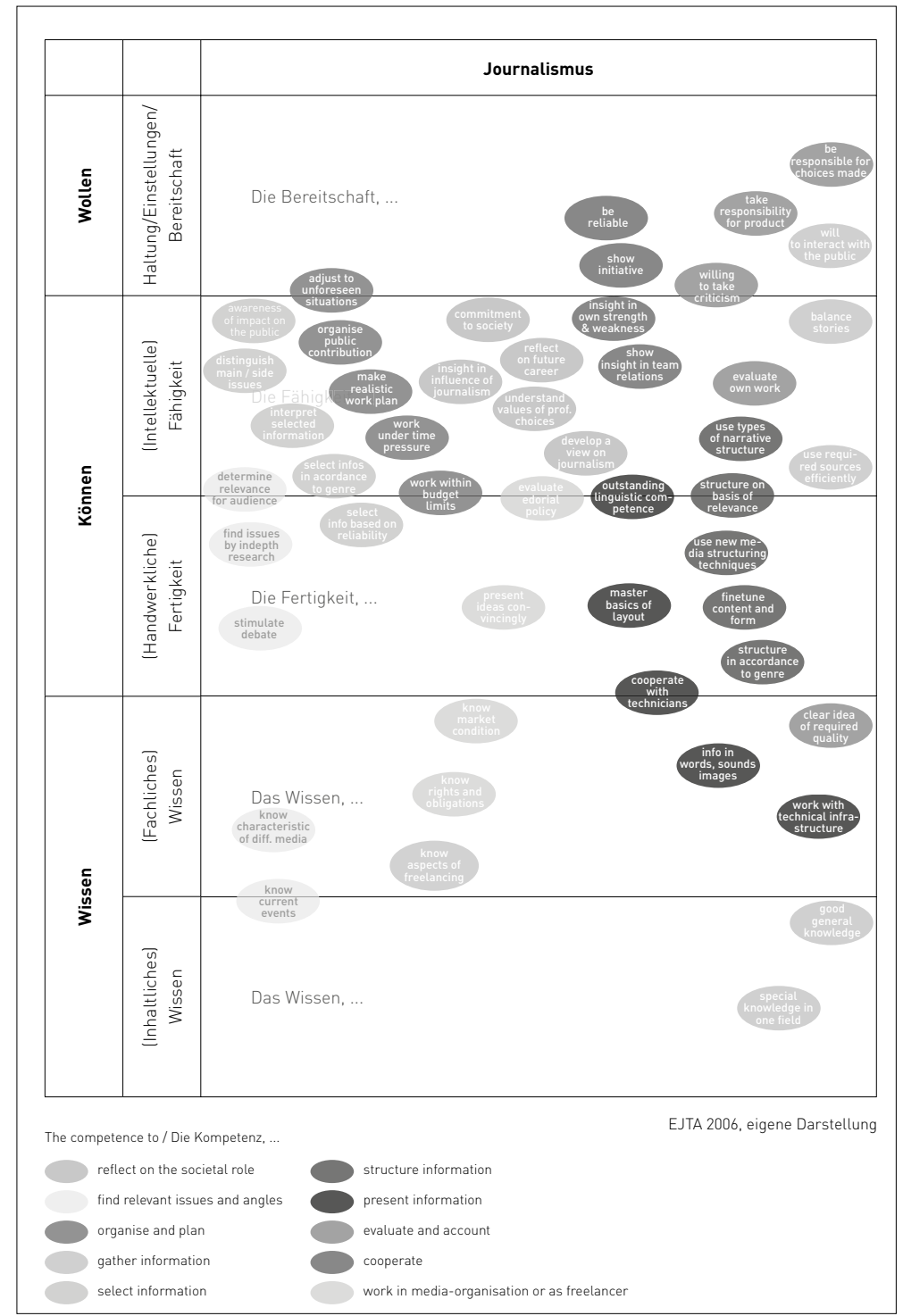

Abb. 2: Die Tartu-Deklaration und die Kompetenz-Matrix nach Stark (2010, 21).

Förderer*innen - und kämpfen, wenn die Spendenmittel knapp werden, mit dem Problem der Refinanzierung ihrer journalistischen Leistungen. 
Auch die Betreiber*innen privatwirtschaftlicher Medienunternehmen - ob Mainstream, in der Nische oder alternativ - sind auf die Refinanzierung der Kosten durch Werbe- und Vertriebserlöse angewiesen. Soweit diese über den Verkauf von Werbezeiten und -flächen erreicht werden soll, zielen sie auf hohe Reichweiten in ihren Zielgruppen und auf Diversifizierung ihrer Dienstleistungen ab (etwa Redakteur*innen als Event-Veranstalter und Touristik-Reiseführer*innen). Nach rund zwei Jahrzehnten des Experimentierens im Online- und Hybridmarkt sehen viele Medienunternehmen, dass ihre Erlösmodelle nicht kostendeckend funktionieren und der Onlinejournalismus trotz seiner Marktanpassung quersubventioniert werden muss. ${ }^{16}$ Die wenigen Ausnahmen - vorneweg die New York Times - dienen nicht als Vorbilder, denn sie nutzen ihre Sonderstellung (die NYT, die Washington Post und The Guardian erreichen mit ihren herausragenden Informationsangeboten den englischsprachigen Raum weltweit als Absatzmarkt) und bestätigen darin ihren Ausnahmecharakter. Auch der Hinweis, dass im deutschsprachigen Raum inzwischen viele Nischenprodukte existieren, die teils als Verein über Abo-Mitgliedschaften, teils dank öffentlicher Zuwendungen oder Fördermittel und Spenden existieren, hilft nicht weiter. Denn diese Medien sind supplementäre Angebote im Dienst der Meinungsvielfalt; sie setzen die kostspielige Informationsleistung der tagesaktuellen Newsmedien voraus. Zudem wirtschaften viele von ihnen mit dem keineswegs beispielgebenden Modell der Selbstausbeutung. Ihre Reichweiten sind gering. Nur wenige dieser Medien - beispielhaft das von der Verlagsgruppe Heise Medien finanzierte Onlinemagazin Telepolis - können jenseits ihrer Community etwas Substanzielles zum gesellschaftlichen Diskurs beitragen.

Der meist betriebswirtschaftlich begründete Personalabbau in vielen Medienredaktionen hat im Laufe der vergangenen zehn Jahre eine bunte Szene an Journalist*innenbüros entstehen lassen (»Media Entrepreneurship «), ${ }^{17}$ die ihre Dienste auf dem Medienmarkt anbieten. Zu ihrem Kompetenzprofil zählt von daher die unspezifische Fertigkeit, Kundenwünsche optimal und effizient zu bedienen. Der

16 Die etablierten Newsmedien - insbesondere von der Süddeutschen Zeitung und der Frankfurter Allgemeinen - meldeten im Herbst 2020 infolge des durch die Corona-Krise ausgelösten Informationsbedarfs einen markant gestiegenen Anteil an Online-Abonnements. Ob dieser zur Refinanzierung genügt und ob er von Dauer sein wird, bleibe dahingestellt.

17 https://www.fachjournalist.de/media-entrepreneuship-wenn-journalisten-unternehmer-werden/. 
Entrepreneur-Trend führte aber auch zur interessanten Überlegung, die Kompetenzen so auszurichten, dass die Journalist*innen kein Medienprodukt, sondern nur Inhalte, also Themen (»Geschichten«) und Berichte bereitstellen. Diese Produkte könnten analog zu Spotify auf einer »anbieterübergreifenden und abonnementbasierten Journalismusplattform « bereitgestellt werden (Wellbrock 2020). Auf diesem Wege, so die Idee, ließe sich der publizistische Wettbewerb und damit die Medien- und Meinungsvielfalt gegen die Web-Monopolisten sichern (ebd.). Der Einwand liegt auf der Hand: Mit diesem Konzept würde der Wettkampf der Solounternehmer*innen um Aufmerksamkeit unterhalb der Medienmarken auf der Mikroebene der Informationsangebote weiter verschärft, mit der Folge eines noch stärkeren Zuschnitts der journalistischen Kompetenzen auf reichweitegenerierende Techniken.

Diese Diskussion führt mich zu der Folgerung, dass der privatwirtschaftlich organisierte Onlinejournalismus ein Gefangener der Vermarktungszwecke und insofern seiner öffentlichen Aufgabe entfremdet bleibt. Und diese Entfremdung wird durch die beschriebene, auf Marktanpassung gerichtete Ausdifferenzierung von Kompetenz weiter verstärkt. So hat es den Anschein, als sei unter den geltenden Bedingungen für den Onlinejournalismus kein Fluchtweg offen: Weder Mitgliedschafts- noch Stiftungs- oder Spendenmodelle generieren die nachhaltig zu sichernden Kapazitäten, um eine gut informierte und diskursiv orchestrierte Medienöffentlichkeit in Gang zu halten. ${ }^{18}$

\section{Zur Rückgewinnung substanzieller Kompetenz: Zwei Perspektiven}

Trotz dieser wenig ermutigenden Bestandsaufnahme bieten die Kommunikations- und Interaktionsräume der Onlinewelt aus meiner Sicht zwei transformatorisch zu deutende Perspektiven, die der Kompetenzdebatte eine neue Richtung geben.

18 Mitunter wird die genossenschaftlich organisierte, sich linksalternativ verstehende tageszeitung (taz) als gelungene Ausnahme genannt. Doch die verkaufte Auflage (Print und E-Paper) erreicht laut IVW knapp 41000 Exemplare. Die minimalen Werbe- und die Vertriebserlöse müssen von den Genossenschaftern bezuschusst werden. Gleichwohl gestatten die Einnahmen keine tarifgemäße Entlohnung der taz-Mitarbeiter*innen. Jedenfalls konnte das taz-Modell unter den Newsmedien nicht als Vorbild dienen. 
Die erste hat das durch die Onlinemedien belebte Projekt »Zivilgesellschaft « im Blick..$^{19}$ Dieses geht davon aus, dass die »Gesellschaftsbürger*innen« quasi unterhalb des Institutionengefüges die Kommunikationsräume durch ihr Bedürfnis, Gestaltungs- und Handlungsziele auszuhandeln, diskursiv beleben (vgl. Habermas 1998, 418-419). Allerdings sind die online interagierenden Individuen, Gruppen und Initiativen selbst nicht in der Lage, die dem Journalismus zugewiesene Informationsfunktion zu erbringen. Indessen können sie sich die Kompetenzen aneignen, die ich zuvor mit dem Leitbild der diskursiven, um Orientierung besorgten Onlinekommunikation umrissen habe. Unter dem Paradigma »Die sich selbst verständigende Zivilgesellschaft « müsste diese Diskurskompetenz aus meiner Sicht als Kulturtechnik vergesellschaftet und in die Bildungsprogramme eingebaut werden vergleichbar der Alphabetisierung der Gesellschaft im Fortgang des 19. Jahrhunderts. Verschiedene Initiativen und Graswurzelprojekte befassen sich derzeit mit diesem Ansatz - auch mein Institut verfolgt unter dem Label »fit for news« ein kleines Pilotprojekt mit dieser Zielstellung. ${ }^{20}$

Die genuin journalistische Kompetenz sollte unter dieser Perspektive auf den Informationsauftrag fokussiert werden: Ihr Kern hätte die mit der öffentlichen Aufgabe verbundene Tätigkeit zum Inhalt, über die relevanten Vorgänge sachrichtig, umfassend und ausgewogen zu informieren und über deren Hintergründe und Handlungszusammenhänge aufzuklären, sprich: Kritik und Kontrolle zu üben. Dies ist weniger trivial, als es erscheinen mag. Studien und Inhaltsanalysen zeigen immer wieder, dass die reichweitestarken Newsmedien der Sichtweise der politischen und wirtschaftlichen Eliten folgen, dass sie komplexe Vorgänge oft einseitig oder parteiergreifend beschreiben, behördliches Versagen wie auch Missstände eher zufällig thematisieren und oftmals statt der nachrichtlichen Aufklärung bevorzugt Meinungs- und Spekulations-

19 Der Begriff ist unscharf und wird in den Sozialwissenschaften auch als Synonym für »Bürger*innengesellschaft« gebraucht (vgl. Zimmer 2012). Ich folge hier Habermas, der »Zivilgesellschaft« als zugangsoffene, freiwillige Kommunikationsgemeinschaft begreift, die auch Formen des gewaltlosen Protestes und zivilen Ungehorsams einschließt. Habermas beschreibt diesen Kommunikationsraum als nicht vermachtete und insofern autochthone Öffentlichkeit. Diesem Raum steht die vermachtete Öffentlichkeit gegenüber, in der die professionellen Mainstreammedien, die politischen Institutionen, Interessengruppen und Lobbyist”innen dominieren (Habermas 1998, 415-467).

20 Näheres unter: https://fitfornews.de/. 
journalismus betreiben. Verkürzt gesagt: Die Kernkompetenz - die unvoreingenommene, auf Offenlegung gerichtete Recherche - findet man nicht regelhaft, sondern ausnahmsweise. Für die deliberativ begründete Diskursöffentlichkeit, die in der zivilgesellschaftlichen Lebenswelt stattfindet, ist diese Informationsaufgabe allerdings die Grundvoraussetzung. Ohne sie gibt es keinen verständigungsorientierten Diskurs, vielmehr Populismus und Propaganda.

$\mathrm{Zu}$ dieser journalistischen Kernkompetenz hinzu tritt die Aufgabe, bedeutsame Konflikte und Probleme zu erkennen, zu analysieren und allgemeinverständlich darzustellen - ich nenne sie hier verkürzt »Thematisierungsfunktion«. Denn im Unterschied zu den 1960er Jahren, als die »öffentliche Aufgabe « erkannt und in den Landespressegesetzen verbindlich beschrieben wurde, sind die heutigen Verhältnisse für viele Menschen unübersichtlich und die Wirkungszusammenhänge zwischen Politik, Wirtschaft und Soziokultur undurchschaubar. Mit anderen Worten: Komplexe Vorgänge und Prozesse in Politik und Wirtschaft zu untersuchen und deren Akteur*innen und Interessen durchsichtig zu machen ist Teil dieser Kompetenz; sie gewinnt seit dem Web 2.0 und der desorientierenden Informationsüberflutung weiter an Bedeutung. Vielleicht gelingt es den solcherart kompetenten Journalist*innen, "sich als Mandatar eines aufgeklärten Publikums [zu] verstehen, dessen Lernbereitschaft und Kritikfähigkeit sie zugleich voraussetzen, beanspruchen und bestärken « (Habermas 1998, 457). Dies steht unter der Prämisse, dass auf Seiten des Publikums - gleichsam als Komplementär - die genannte Medienkompetenz erworben werde.

Wie aber sähe die mediale Infrastruktur aus, die diesen gesellschaftlichen Diskurs in Gang halten könnte? Hier setzt meine zweite Perspektive an. Sie greift eine Debatte auf, die vor mehr als 70 Jahren in der Gründungsphase der Bundesrepublik Deutschland schon einmal in Fahrt kam, als es um die Bedingungen eines Journalismus ging, der aus ökonomisch und ideologisch unabhängiger Position informieren kann. Es war die Idee des public service, mithin eines meritorischen Dienstes, vergleichbar dem Gesundheits- und Bildungssystem, die eigentlich nicht marktfähig sind (»öffentliche Güter«; Kiefer 2001, 132-133) und deshalb nicht kommerzialisiert, sondern als gemeinnützige oder öffentlich-rechtliche Einrichtungen zu organisieren wären. Jedenfalls sollte der Informationsjournalismus von keinem Finanzier, keiner Machtgruppe, keiner politischen Partei und keiner gewinnorientierten Eigentümerin abhängig sein. Dies schien realisierbar, sofern diese Medien - ähnlich wie die BBC in Großbritannien - allein über Gebühren 
finanziert und von gesellschaftlich relevanten Gruppen kontrolliert würden (über Organisation und Einflussnahme der Regierung vermittels des BBC Trust vgl. Hahn 2010, 136; inzwischen wurde der BBC Trust durch Ofcom ersetzt).

In Deutschland ist diese Idee so alt wie der vom britischen Journalisten Hugh Carleton Greene im Oktober 1946 gegründete Nordwestdeutsche Rundfunk (NWDR), das Urmodell der ARD: »Unerbittlich in der Abwehr von äußerem Einfluss, aber auch unerbittlich für Toleranz«, nannte Greene (1970) sein Credo. Auf der Suche nach einer Organisationsform, die dieses Prinzip Unabhängigkeit sichern sollte, fand er das Konzept der öffentlich-rechtlichen Einrichtung: Finanzielle Unabhängigkeit gegenüber Verbänden und Werbewirtschaft, also keine Werbung, keine Steuergelder, sondern einzig Zuschauerbeiträge. Zudem politische Unabhängigkeit gegenüber dem Staat und den Parteien, also kein Weisungsrecht der Länderregierungen, vielmehr ein pluralistisch besetzter Rundfunkrat (damals Hauptausschuss), der relevante gesellschaftliche Interessen repräsentiert und der den Intendanten (damals Generaldirektor) wählt.

Greenes Überzeugung, dass in einer Demokratie die Meinungsbildung der Bürger*innen ohne staatliche Einflussnahme allein auf die Informations- und Kommentarleistung unabhängiger Journalist*innen abgestützt sein sollte, fanden sogar die US-amerikanischen Besatzer richtig. In ihrer Anordnung vom 21. November 1947 hieß es: »Es ist die grundlegende Politik der US-Militärregierung, dass [...] die Mittel der öffentlichen Meinungsbildung wie Presse und Rundfunk [...] von jeder Regierungseinwirkung freigehalten werden.«

Es blieben Erinnerungen an die Zukunft des kompetenten Informationsjournalismus, denn bereits im Frühjahr 1948 hatten die deutschen Politiker Greenes Konzept demoliert: Statt unabhängiger Leute aus dem Bildungswesen setzten sich die Kultusminister der vier Länder gleich selbst in den Hauptausschuss und zogen ihre Leute nach. Seither wurde der Einfluss der Politik auf die Rundfunkanstalten nicht ab-, sondern ausgebaut - in den Anstalten der ARD ebenso wie 15 Jahre später im ZDF. Eindrücklich zeigt dies die Causa Brender: Der ZDF-Chefredakteur galt den Unionspolitiker*innen als zu kritisch und politisch zu links, weshalb der von ihnen dominierte Verwaltungsrat Ende 2009 Brenders Vertragsverlängerung ablehnte. Ihre Macht schrumpfte ein wenig, als das Bundesverfassungsgericht 2014 die Zusammensetzung des Verwaltungsrats als rechtswidrig einstufte. Seither werden nur mehr rund ein Drittel der Mitglieder direkt oder indirekt von politischen Parteien und 
Ämtern delegiert. Doch dies genügt. ${ }^{21}$ So sind bis heute die leitenden Journalist*innen der ARD und des ZDF den politisch besetzten Aufsichtsgremien gegenüber rechenschaftspflichtig. Viele Vorfälle belegen, dass keine der beiden Anstalten de facto die Staatsferne aufweist, die ihnen 1947 per Proklamation zugedacht worden war.

Jener Grundsatz der Staats- und Wirtschaftsferne könnte heute wiederbelebt werden, zumal es nicht mehr um die Hoheit über wenige Sendefrequenzen geht, sondern offene Kommunikationsräume im Internet zur Verfügung stehen. Sofern die Zivilgesellschaft ihr Diskursinteresse artikulieren kann, müssten ihre Akteur*innen darauf bestehen, den Informationsjournalismus an die öffentliche Aufgabe zu binden und im Internet als offene Plattform des public service zu organisieren. Dabei müsste die Staatsferne organisationsrechtlich, die ökonomische Eigenständigkeit über Beiträge abgesichert, die medienrechtliche Qualitätskontrolle institutionalisiert und von medienkompetenter Seite supervisioniert werden.

Vorgänge im Ausland zeigen, dass diese Idee verstanden und vom Publikum gewünscht wird. Zum Beispiel die dreisprachige Schweizerische Radio- und Fernsehgesellschaft (SRF): Sie definiert sich als »service public«, ist als Verein organisiert, erhält von der Bundesregierung die Konzession und wird vom Bundesamt für Kommunikation nur in formaler Hinsicht (Einhaltung der Rechtsbestimmungen) kontrolliert. Für die programminhaltliche »Beurteilung (nicht Kontrolle) ist ein Publikumsrat zuständig, dessen 26 Mitglieder verschiedene Bevölkerungsgruppen repräsentieren - ein sich zivilgesellschaftlich legitimierendes Gremium. ${ }^{22}$ Außerdem prüfen in den Sprachregionen OmbudsmannEinrichtungen alle Publikumsbeschwerden; Beschwerdeführer*innen

21 Eine Zusammenstellung markanter Einflussnahmen der Politik auf die journalistische Aussagenproduktion der öffentlich-rechtlichen Anstalten brachte die Süddeutsche Zeitung am 25.10.2012, https://www.sueddeutsche.de/medien/ politik-und-medien-wenn-der-minister-zweimal-klingelt-1.1505696.

22 Aus seiner Selbstdarstellung: »Als wichtiges Glied der Qualitätskontrolle gibt er den Programmverantwortlichen und Programmschaffenden von SRF kritische und konstruktive Anregungen aus der Sicht der Konsumentinnen und Konsumenten von SRF-Programmen. Der Publikumsrat ist unabhängig und nicht weisungsbefugt. Er beobachtet ausgewählte Programme, Sendungen und Online-Angebote von SRF und 3sat nach eigener Wahl. Die Programmbeobachtung wird in ständigen Arbeitsgruppen geleistet. Die Ergebnisse der Arbeitsgruppen werden im Plenum mit den Sendeverantwortlichen diskutiert und anschliessend über die verschiedenen Online- und Offlinekanäle der SRG.D öffentlich gemacht.«https://www.srgd.ch/de/uber-uns/publikumsrat/ueber-uns/. 
können ihren Fall an die »Unabhängige Beschwerdeinstanz« weiterziehen. Während in Deutschland mit seiner zehnfach größeren Bevölkerung in allen Gremien und Beiräten der ARD und des ZDF insgesamt rund 1000 Vertreter*innen von Gruppen, Einrichtungen und Parteien tätig sind, engagieren sich in den Mitglieds- und Regionalgesellschaften der SRG rund 23000 Personen (Siepmann 2012). Im März 2018 hat die große Mehrheit der Schweizer Stimmbürger*innen eine Initiative zur Abschaffung der Rundfunkgebühren rundheraus abgelehnt. Den Bezeugungen zufolge war dies, zivilgesellschaftlich betrachtet, die vielleicht deutlichste Bestätigung weniger der Unterhaltungsleistung als der journalistischen Informations- und Orientierungsfunktion zumal im Zeitalter von YouTube und Instagram.

Abschließend ziehe ich aus den skizzierten Perspektiven einige konkrete Folgerungen. Ich formuliere sie auf der Handlungsebene als Bedingungen für eine Erneuerung der Kompetenzen, die Informationsjournalist*innen in der Ära des partizipatorischen, über Vernetzungen strukturierten, dabei von intermediären Konzernen bemächtigten Internets benötigen:

- Die zivilgesellschaftliche Lebenswelt: Diskursräume zurückholen, öffentlich-rechtlich absichern und dekommerzialisieren.

- Die organisierte Mediengesellschaft: Die Kulturtechnik »Medienund Informationskompetenz « als Basisbildungsprogramm einführen, vergleichbar seinerzeit mit der Alphabetisierung.

- Das Institutionengefüge des politischen Systems: Verrechtlichter Schutz der Kommunikationsräume vor staatlicher beziehungsweise parteipolitischer Einflussnahme (Neutralitätsschutz) sowie kartellrechtliche Abwehr der Monopolisierungstendenzen.

- Die Informationsstruktur als meritorischen public service des »Informationsjournalismus « organisieren und über ein macht- und interessenunabhängiges Modell (etwa Stiftungen, Beiträge) finanzieren.

- Die Handhabung journalistischer Kompetenz durch die angewandte Kommunikations- und Medienwissenschaft periodisch evaluieren und deren Befunde den für die Qualitätskontrolle geschaffenen Gremien auf der Ebene der Zivilgesellschaft an die Hand geben. 


\section{Literatur}

Becker, Sabine, und Markus Kaiser (Hrsg.). 2014. Berufe in den Medien. München: Verlag Dr. Gabriele Hooffacker.

Bönisch, Julia. 2019. Wir brauchen gute Manager an der Spitze von Redaktionen. Journalist 5: 60-64.

Dernbach, Beatrice; Wiebke Loosen (Hrsg.) 2012. Didaktik der Journalistik. Konzepte, Methoden und Beispiele aus der Journalistenausbildung. Wiesbaden: Springer VS.

Deutscher Journalisten-Verband. 2003. Memorandum: Journalistische Aus- und Weiterbildung in der Krise. Beschlossen auf dem DJV-Verbandstag 2003 in Wiesbaden. Bonn: DJV.

Donsbach, Wolfgang.1982. Legitimationsprobleme des Journalismus. Gesellschaftliche Rolle der Massenmedien und berufliche Einstellung von Journalisten. Freiburg im Breisgau: Verlag Karl Alber.

Donsbach, Wolfgang. 1999. Journalismus und journalistisches Berufsverständnis. In: Mediengeschichte der Bundesrepublik Deutschland, herausgegeben von Jürgen Wilke, 489-517. Köln: Böhlau.

Donsbach, Wolfgang. 2012. Hausaufgaben noch immer nicht gemacht: Versäumnisse und Konzepte der Journalistenausbildung. In: Didaktik der Journalistik. Konzepte, Methoden und Beispiele aus der Journalistenausbildung, herausgegeben von Beatrice Dernbach und Wiebke Loosen, 31-44. Wiesbaden: Springer VS.

Elmer, Christina, Stefan Ottlitz, und Matthias Streitz. 2020. Wie wir den Spiegel entwickeln. Journalist 3: 56-60.

Fabris, Hans Heinz. 1971. Das Selbstbild des Kommunikators bei Tageszeitungen. Publizistik 16 (4): 357-368.

Greene, Hugh Carleton. 1970. Entscheidung und Verantwortung - Perspektiven des Rundfunks. Hamburg: Hans-Bredow-Institut.

Habermas, Jürgen. 1998. Faktizität und Geltung. Beiträge zur Diskurstheorie des Rechts und des demokratischen Rechtsstaates. Frankfurt am Main: Suhrkamp.

Hahn, Caroline. 2010. Die Aufsicht des öffentlich-rechtlichen Rundfunks. Bestandsaufnahme und Zukunftsperspektiven. Frankfurt am Main: Peter Lang.

Haller, Michael. 2000. Die zwei Kulturen. Journalismustheorie und journalistische Praxis. In: Theorien des Journalismus. Ein diskursives Handbuch, herausgegeben von Martin Löffelholz, 101-122. Wiesbaden: Westdeutscher Verlag.

Haller, Michael. 2012. Didaktischer Etikettenschwindel? Die Theorie-Praxis-Verzahnung in der Journalistenausbildung. In: Didaktik der Journalistik. Konzepte, Methoden und Beispiele aus der Journalistenausbildung, herausgegeben von Beatrice Dernbach und Wiebke Loosen, 45-58. Wiesbaden: Springer VS.

Harnischmacher, Michael. 2010. Journalistenausbildung im Umbruch. Zwischen Medienwandel und Hochschulreform: Deutschland und USA im Vergleich. Konstanz: UVK.

Holzer, Horst. 1973. Kommunikationssoziologie. Reinbek bei Hamburg: Rowohlt. Hömberg, Walter (Hrsg.). 1978. Journalistenausbildung. Modelle, Erfahrungen, Analysen. München: Ölschläger. 
Kiefer, Marie Luise. 2001. Medienökonomik. Einführung in eine ökonomische Theorie der Medien. München: Oldenbourg.

Kolo, Castulus, Thomas Döbler, und Lars Rademacher. 2012. Wertschöpfung und Medien. Versuch einer Einordnung von Perspektiven, Konzepten und Begrifflichkeit. In: Wertschöpfung durch Medien im Wandel, herausgegeben von Castulus Kolo, Thomas Döbler und Lars Rademacher, 9-18. Baden-Baden: Nomos.

Koszyk, Kurt. 1974. Professionalisierung durch Wissenschaft. Journalistenausbildung zwischen Berufung und Beruf. Aus Politik und Zeitgeschichte 24: 27-37.

Langenbucher, Wolfgang. 1974. Zukünftige Erfordernisse für die Ausbildung der Journalisten. In: Massenmedien in der Prognose. Konzepte und Modelle für die Zukunft, herausgegeben von Uwe Magnus, 150-156. Berlin: Spiess.

Meier, Klaus. 2007. Journalistik. Konstanz: UVK.

Merten, Klaus. 1994. Konvergenz der deutschen Fernsehprogramme: eine Langzeituntersuchung 1980-1993. Münster, Hamburg: Lit.

Neuberger, Christoph, Christian Nuernbergk, und Melanie Rischke (Hrsg.). 2009. Journalismus im Internet. Profession - Partizipation - Technisierung. Wiesbaden: Springer VS.

Neuberger, Christoph. 2002. Online-Journalismus: Akteure, redaktionelle Strukturen und Berufskontext. Medien \& Kommunikationswissenschaft 50 (1): 102114.

o.A. 2019. Trennung von Redaktion und Verlag infrage gestellt: SZ-Digitalchefin Julia Bönisch hat Ärger im eigenen Haus. Meedia.de vom 14. Mai, https://meedia.de/2019/05/14/aerger-um-gastbeitrag-sz-digitalchefin-julia-boenischmusste-sich-vor-kollegen-rechtfertigen/. Zugegriffen: 3. Dezember 2020.

Oswald, Bernd. 2013. Vom Produkt zum Prozess. In: Journalismus in der digitalen Moderne. Einsichten - Ansichten - Aussichten, herausgegeben von Leif Kramp, Leonard Novy, Dennis Ballwieder und Karsten Wenzlaff, 63-79. Wiesbaden: Springer VS.

Plöchinger, Stefan. 2013. »Mauern passen nicht zum Netz«. Interview mit dem Süddeutsche.de-Chef. T3n.de vom 27. Januar, https://t3n.de/magazin/suddeutschede-chef-stefan-plochinger-interview-mauern-231149/. Zugegriffen: 3. Dezember 2020.

Pörksen, Bernhard. 2018. Die große Gereiztheit. Wege aus der kollektiven Erregung. München: Hanser.

Quandt, Thorsten. 2005. Journalisten im Netz. Eine Untersuchung journalistischen Handelns in Online-Redaktionen. Wiesbaden: Springer VS.

Rühl, Manfred. 1980. Journalismus und Gesellschaft. Bestandsaufnahme und Theorieentwurf. Mainz: Hase \& Koehler.

Ruß-Mohl, Stephan. 2017. Die informierte Gesellschaft und ihre Feinde. Warum die Digitalisierung die Demokratie gefährdet. Köln: Herbert von Halem.

Schatz, Heribert. 1994. Rundfunkentwicklung im »dualen System«: die Konvergenzhypothese. In: Politische Kommunikation in Hörfunk und Fernsehen. Elektronische Medien in der Bundesrepublik Deutschland, herausgegeben von Otfried Jarren, 67-80. Opladen: Westdeutscher Verlag.

Schätz, Konstantin, und Susanne Kirchhoff. 2020. Neue Wege im Journalismus, 
Weichenstellung in der Ausbildung. Journalistik 3 (2): 98-110. https://journalistik.online/ausgabe-2-2020/neue-wege-im-journalismus-weichenstellung-inder-ausbildung/.

Schmolke, Michael. 1970. Die deutschen Journalisten der Nachkriegszeit in der Selbstdarstellung ihrer Standespresse. In: Publizistik zwischen Intuition und Gewißheit: Drei Vorträge, herausgegeben vom Institut für Publizistik Münster, 4161. Assen: van Gorkum.

Siepmann, Ralf. 2012. Konvergenz auf eidgenössische Art. Die SRG SSR treibt den Transfer ins digitale Zeitalter voran. epd medien Nr. 28 vom 13. Juli.

Skriver, Ansgar. 1970. Schreiben und schreiben lassen. Innere Pressefreiheit - Redaktionsstatute. Karlsruhe: Müller.

Stark, Alexandra. 2010. Journalism Reloaded - Was Journalistinnen und Journalisten für die Zukunft lernen müssen. Masterarbeit. Leipzig School of Media und Medienausbildungszentrum Luzern. https://www.leipzigschoolofmedia.de/ hubfs/6494216/Masterarbeiten/Masterarbeit_Stark-Alexandra_2010.pdf. Zugegriffen: 3. Dezember 2020.

Weischenberg, Siegfried. 1990. Journalismus und Kompetenz. Qualifizierung und Rekrutierung für Medienberufe. Opladen: Westdeutscher Verlag.

Weischenberg, Siegfried, Klaus-Dieter Altmeppen, und Martin Löffelholz (Hrsg.). 1994. Die Zukunft des Journalismus, Technologische, ökonomische und redaktionelle Trends. Opladen: Westdeutscher Verlag.

Wellbrock, Christian-Mathias. 2020. »Spotify für Journalismus«, »Verlagsplattform«, »Digitales Pressegrosso« - Drei Szenarien für eine anbieterübergreifende Journalismusplattform. Journalistik 3 (2): 131-149. https://journalistik.online/ausgabe-2-2020/spotify-fuer-journalismus-verlagsplattform-digitalespressegrosso/. Zugegriffen: 3. Dezember 2020.

Zeuner, Bodo. 1973. Zur Standesideologie der Journalisten. In: Solidarität gegen Abhängigkeit. Auf dem Weg zur Mediengewerkschaft, herausgegeben von Ulrich Paetzold und Hendrik Schmidt, 13-36. Darmstadt: Luchterhand.

Zimmer, Annette. 2012. Die verschiedenen Dimensionen der Zivilgesellschaft. In: Dossier Deutsche Verhältnisse. Eine Sozialkunde, herausgegeben von der Bundeszentrale für politische Bildung. https://www.bpb.de/politik/grundfragen/ deutsche-verhaeltnisse-eine-sozialkunde/138713/dimensionen. Zugegriffen: 3. Dezember 2020.

\section{Open Access}

Dieser Beitrag erscheint unter der Creative-Commons-Lizenz CC BY-ND 3.0 DE: https://creativecommons.org/licenses/by-nd/3.0/de/. 